\title{
A Novel Position-Sensitive Radiation Detector Using a Gaseous Photomultiplier and a UV Scintillator
}

\author{
Shunsuke Kurosawa ${ }^{1}$ \\ Department of Physics, Graduate School of Science, Kyoto University \\ Kyoto 606-8502, Japan \\ E-mail: kurosawa@cr.scphys.kyoto-u.ac.jp \\ Hidetoshi Kubo, Toru Tanimori, Kojiro Taniue \\ Department of Physics, Graduate School of Science, Kyoto University \\ Kitashirakawa-Oiwake-cho, Sakyo-ku, Kyoto 606-8502, Japan
}

\section{Hiroyuki Sekiya}

Kamioka Observatory, Institute for Cosmic Ray Research, University of Tokyo, 456, Higashi-Mozumi Hida, Gifu 506-1205, Japan

Kamioka Satellite,Institute for the Physics and Mathematics of the Universe, 456, Higashi-Mozumi Hida, Gifu 506-1205, Japan

\section{Akira Yoshikawa, Takayuki Yanagida, Yuui Yokota \\ Institute of Multidisciplinary, Research for Advanced Materials, Tohoku University, \\ 2-1-1, Katahira, Aoba, Sendai, Miyagi 980-8577, Japan}

Kentaro Fukuda, Sumito Ishizu, Noriaki Kawaguchi, Toshihisa Suyama

Tokuyama Corporation

3-3-1, Shibuya, Shibuya, Tokyo 150-8383, Japan

\begin{abstract}
We developed a novel position-sensitive radiation detector that consists of an ultraviolet (UV) imaging gas photomultiplier and a UV scintillator. The former consists of a micro pixel gaseous chamber ( $\mu$-PIC), two gas electron multipliers (GEMs), and a semitransparent CsI photocathode of diameter $34 \mathrm{~mm}$ deposited on a $\mathrm{MgF}_{2}$ window. It was coupled to a $\mathrm{LaF}_{3}(\mathrm{Nd})$ scintillator that emits UV light. We successfully conducted photon counting and imaging using this detector along with an alpha particle source.
\end{abstract}

International Workshop on New Photon Detectors (PD09)

Shinshu University, Matsumoto, Japan

24-26 June 2009

$1 \quad$ Speaker 


\section{Introduction}

Vacuum photomultiplier tubes (PMTs) are used in various fields such as high-energy physics [1], medical imaging [2], and gamma-ray astronomy [3]. Because a flat, large-area PMT cannot be produced easily, a large number of PMTs are necessary in applications that need large-area coverage. On the other hand, a gas photomultiplier (GPM) $[4,5]$ with both a photocathode and a structure for gas avalanche multiplication can be made with a very large area, because large-area micropattern gaseous detectors such as a Micromegas [6], gas electron multiplier (GEM) [7-9], and micro pixel gaseous chamber ( $\mu$-PIC) $[10,11]$ have been operated. Some groups developed ultraviolet- (UV-) or visible-sensitive GPMs with CsI or bialkali photocathodes, respectively $[4,5,12]$. Although the bialkali photocathode has extremely strong chemical reactions, the CsI photocathode is easier to handle.

A UV imaging detector with a CsI photocathode can be applied to material analysis research and liquid $\mathrm{Ar} / \mathrm{Xe}$ scintillators. Furthermore, in combination with UV scintillating crystals, it can be a hard X-ray imaging device that compensates for the low detection efficiency of gaseous detectors. Some groups reported UV scintillators with rapid decay times [13, 14], and $\mathrm{Nd}^{3+}$ doped $\mathrm{LaF}_{3}$ was investigated for gas detectors [15]. Thus, we developed CsI-based position-sensitive GPMs combined with a $\mathrm{LaF}_{3}(\mathrm{Nd}) \mathrm{UV}$ scintillator. In this paper, we describe the photon-counting performance and imaging capability of such a device.

\section{UV scintillator}

We developed a $\mathrm{LaF}_{3}(\mathrm{Nd})$ crystal doped with $8 \% \mathrm{~mol} \mathrm{Nd}^{3+}$ that is $15 \mathrm{~mm} \times 15 \mathrm{~mm} \times 15$ $\mathrm{mm}$ in size. We wrapped the $\mathrm{LaF}_{3}(\mathrm{Nd})$ in GORE-TEX $^{\mathrm{TM}}$ as a reflector, and then coupled it to a UV-sensitive PMT (Hamamatsu R8778) with UV-transparent grease $\left(\operatorname{Krytox}^{\mathrm{TM}}\right)$ to measure the light output of the $\mathrm{LaF}_{3}(\mathrm{Nd})$ irradiated with $5.5 \mathrm{MeV} \alpha$ particles from a 3 $\mathrm{kBq}{ }^{241} \mathrm{Am}$ source.

Figure 1 shows the light output spectrum of the $\mathrm{LaF}_{3}(\mathrm{Nd})$ with a peak of 33 photoelectrons. Because scintillation emission of $\mathrm{LaF}_{3}(\mathrm{Nd})$ lies around $172 \mathrm{~nm}$, and the quantum efficiency of the R8778 at $172 \mathrm{~nm}$ is about $30 \%$ [16], the photon yield of the $\mathrm{LaF}_{3}(\mathrm{Nd})$ was found to be approximately 100 photons $/ 5.5 \mathrm{MeV} \alpha$.

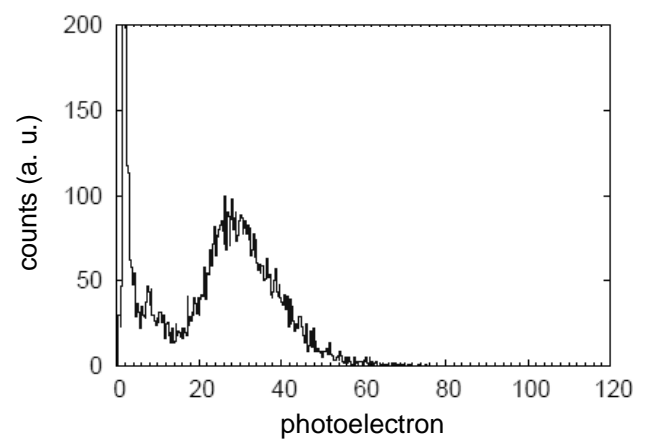

Figure 1: Photoelectron spectrum of $\mathrm{LaF}_{3}(\mathrm{Nd})$ irradiated with $5.5 \mathrm{MeV} \alpha$ particles from an ${ }^{241} \mathrm{Am}$ source. 


\section{Photon counting with GPM and $\mathrm{LaF}_{3}(\mathrm{Nd})$}

\subsection{Setup}

We demonstrated photon counting and evaluated the quantum efficiency using our GPM, which consists of a $\mu$-PIC and two GEMs that suppress the avalanche-induced photon and ion feedback and provide high-gain operation (Fig. 2). The GEMs are $10 \mathrm{~cm} \times 10 \mathrm{~cm}$ in size with holes that are $70 \mu \mathrm{m}$ in diameter and $140 \mu \mathrm{m}$ in pitch, and the insulator is a liquid crystal polymer $100 \mu \mathrm{m}$ thick. The $\mu$-PIC is $10 \mathrm{~cm} \times 10 \mathrm{~cm}$ in size with 256 anode strips and 256 cathode strips, which are placed orthogonally on both sides of a polyimide substrate with a pitch of $400 \mu \mathrm{m}$ [11]. The separation between the second GEM and the $\mu$-PIC is $6 \mathrm{~mm}$; and that between the two GEMs is $2 \mathrm{~mm}$.

The $\mathrm{MgF}_{2}$ window of the GPM is $54 \mathrm{~mm}$ in diameter and $5 \mathrm{~mm}$ thick. A semitransparent CsI photocathode was evaporated and deposited on the $\mathrm{MgF}_{2}$ window with a thickness of the order $10 \mathrm{~nm}$ and an effective area $34 \mathrm{~mm}$ in diameter. To make a drift region, an aluminum layer was also evaporated along the outer $10 \mathrm{~mm}$ of the window's surface. A high drift voltage was supplied to the aluminum electrode through the contact with a 1-mm-thick copper ring. Between the photocathode and the first GEM, which were separated by a distance of $13 \mathrm{~mm}$, a guard ring was placed to make a uniform electric field and to drift photoelectrons to the first GEM. The gas volume was enclosed in a sealed steel vessel and filled with an argon-ethane gas mixture (9:1) at a pressure of $1 \mathrm{~atm}$.

\subsection{Response to UV light}

$\mathrm{An}^{241} \mathrm{Am}$ source and the $\mathrm{LaF}_{3}(\mathrm{Nd})$ (as the light source) were coupled to the $\mathrm{MgF}_{2}$ window with Krytox $^{\mathrm{TM}}$ grease. The $\mu$-PIC was driven at $465 \mathrm{~V}$, and the voltage across the GEM was 422 $\mathrm{V}$. The total gas gain was approximately $2.6 \times 10^{5}$. Each readout channel (sum of 64 strips of the $\mu$-PIC) was amplified to $0.1 \mathrm{~V} / \mathrm{pC}$. The typical pulse height of the amplified cathode signal was approximately $5 \mathrm{mV}$, and its calculated number of photoelectrons was approximately 1 from the gas gain. Figure 3 shows the obtained spectrum. The gas gain of the detector is high enough to have sensitivity to detect single photoelectron. Because the intensity of the light source is 100 photons, the quantum efficiency of this CsI photocathode is approximately $1 \%$.

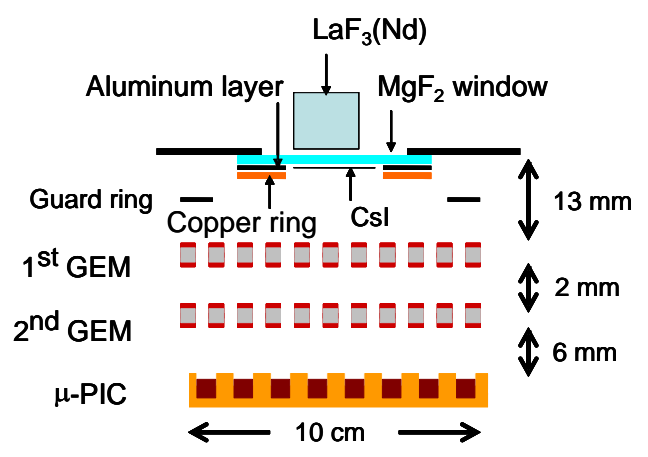

Figure 2: Schematic view of the detector for photon counting.

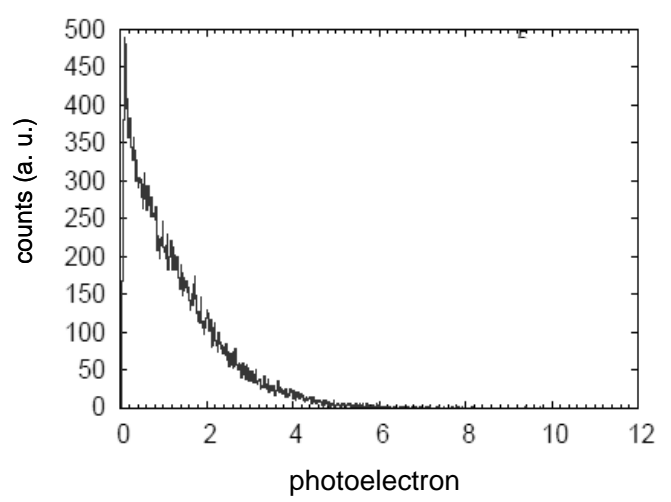

Figure 3: Photoelectron spectrum of UV gaseous detector coupled to $\mathrm{LaF}_{3}(\mathrm{Nd})$ irradiated with $5.5 \mathrm{MeV} \alpha$ particles from an ${ }^{241} \mathrm{Am}$ source. 


\section{Imaging with GPM and $\mathrm{LaF}_{3}(\mathrm{Nd})$}

\subsection{Setup}

We demonstrated imaging using our GPM with a $\mu$-PIC readout system [17]. The configuration was the same as the one shown in Fig. 2, except for the following points: (i) The insulator for the GEMs was made of Kapton ${ }^{\mathrm{TM}}$ polyimide, (ii) the GEMs were $50 \mu \mathrm{m}$ thick, and (iii) the distances between the $\mu$-PIC, GEM, and photocathodes were those shown in Fig 4. The gas volume was enclosed in a sealed aluminum vessel, and filled with an argon-ethane gas mixture $(9: 1)$ at a pressure of $1 \mathrm{~atm}$. The drift and induction fields were set to $0.5 \mathrm{kV} / \mathrm{cm}$ and $2.95 \mathrm{kV} / \mathrm{cm}$, respectively. We measured the gain as a function of the GEM voltage, as shown in Fig. 5. When we obtained an image, the anode voltage of the $\mu$-PIC was $490 \mathrm{~V}$, and the voltage across the GEM was $280 \mathrm{~V}$. The detector was operated with a total gas gain of $6.7 \times 10^{5}$ (the gas gain of the two GEMs was approximately 100).

The output charges from the anode and cathode channels of the $\mu$-PIC were pre-amplified $(0.7 \mathrm{~V} / \mathrm{pC})$, shaped with a gain of 7 , and discriminated via amplifier-shaper-discriminator (ASD) chips (four channels/chip, SONY CXA3653Q) [18]. All of the discriminated digital signals were sent to a position-encoding module based on field programmable gate arrays (FPGAs) with an internal clock of $100 \mathrm{MHz}$, so that the anode and cathode coincident positions $(\mathrm{X}, \mathrm{Y})$ were reconstructed in a memory module.

\subsection{Imaging}

We tested the imaging performance using a $\mathrm{LaF}_{3}(\mathrm{Nd})$ crystal $18 \mathrm{~mm} \times 21 \mathrm{~mm} \times 20 \mathrm{~mm}$ in size. One face $18 \mathrm{~mm} \times 21 \mathrm{~mm}$ in size was coupled to the $\mathrm{MgF}_{2}$ window, and the opposite face was irradiated with $5.5 \mathrm{MeV} \alpha$ particles from a $2.6 \mathrm{MBq}{ }^{241} \mathrm{Am}$ source. Although the crystal was wrapped in GORE-TEX ${ }^{\mathrm{TM}}$ as a reflector, only the $5 \mathrm{~mm} \times 5 \mathrm{~mm}$ area in the center of one face was open to irradiation by $\alpha$ particles.

The crystal was placed on the window in two different positions, one with the edge at the top and the other with the corner at the top and attached with Krytox ${ }^{\mathrm{TM}}$ grease; images were

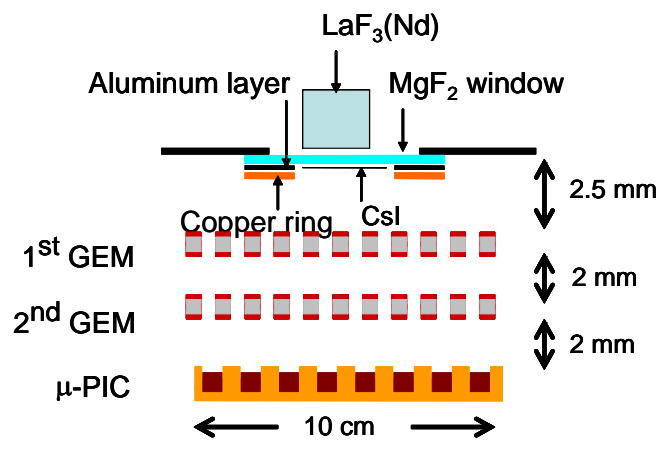

Figure 4: Schematic view of the detector for imaging.

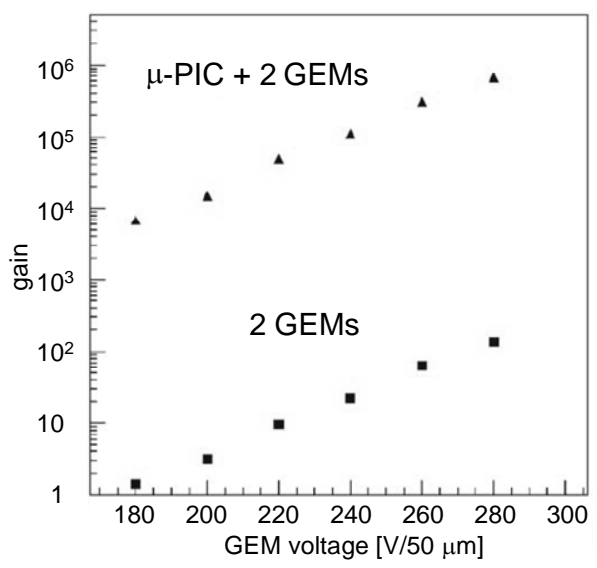

Figure 5: Gas gain as a function of voltage across the GEM. Triangles and squares denote total gain and the gain of the two GEMs, respectively. 
(a)

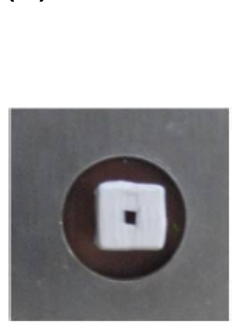

(c)

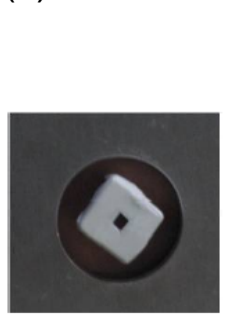

(b)

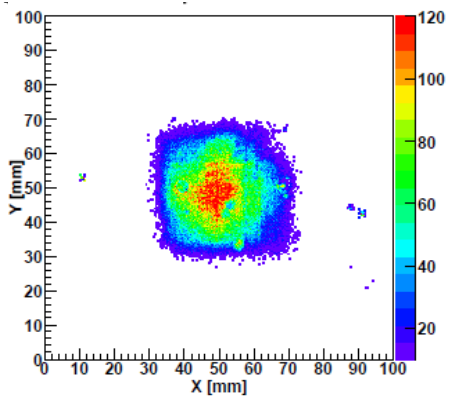

(d)

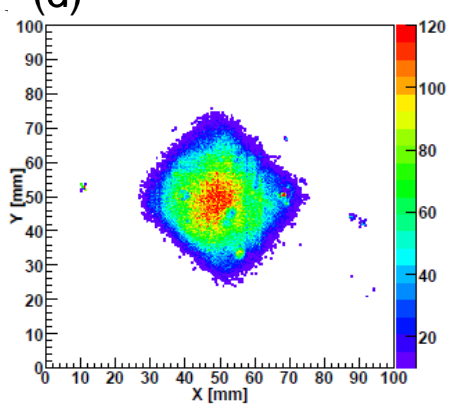

Figure 6: Photographs of $\mathrm{LaF}_{3}(\mathrm{Nd})$ on the window are shown in (a) and (c); (b) and (d) are the respective images. (a)

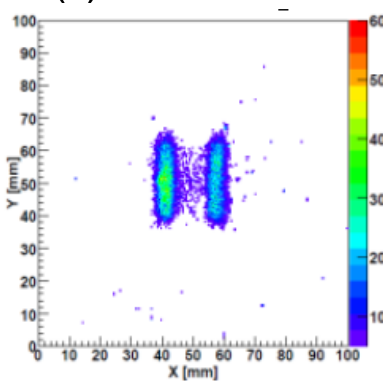

(b)

(c)

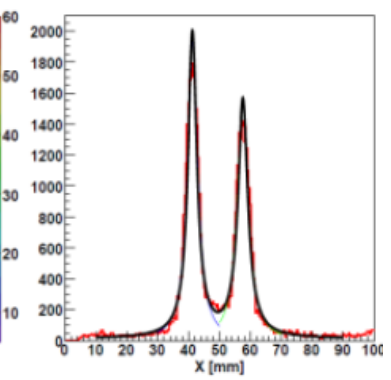

(d)

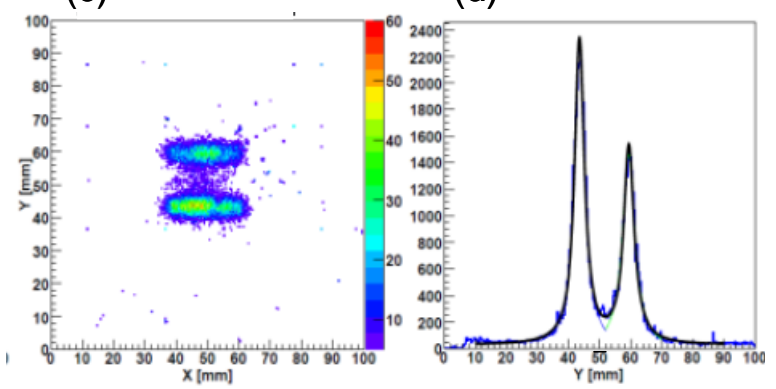

Figure 7: Images of the slits (a, c) and their projections to the $\mathrm{X} / \mathrm{Y}$ axis $(\mathrm{b}, \mathrm{d})$. Double Lorentzian functions are fitted to the projections.

taken as shown in Fig. 6. Incidentally, some hot spots and cold spots appear in both images at the same position. These are caused by the non-uniformity of the photocathode and noise in the readout circuits. Although the scale is larger than the actual size, the images show the shape of the crystal clearly.

To evaluate the magnification and position sensitivity, we used a polyvinyl chloride mask $100 \mu \mathrm{m}$ thick, which had two slits, each $2 \mathrm{~mm} \times 15 \mathrm{~mm}$ in size, separated by $10 \mathrm{~mm}$. This mask was inserted between the crystal and the window, and we obtained the masked images (Fig. 7). From the $\mathrm{X}$ and $\mathrm{Y}$ projection plots shown in Fig. 7, the images are magnified uniformly with a magnification of 1.6.

\section{Conclusions}

We developed a new UV GPM based on a CsI photocathode, GEMs, and $\mu$-PIC. Although the quantum efficiency of the semitransparent CsI photocathode is still limited, the detector showed good single-photoelectron sensitivity. Furthermore, we succeeded in demonstrating imaging using UV GPM and a $\mathrm{LaF}_{3}(\mathrm{Nd})$ crystal. In this measurement, newly developed $\mathrm{LaF}_{3}(\mathrm{Nd})$ scintillators were used as UV light sources. A combination of such fluoride UV scintillators and imaging GPMs can be novel radiation imaging detectors that compensate for the low detection efficiency of gas detectors. 


\section{References}

[1] R. Dzhelyadin et al., The LHCb calorimeter detectors, Nucl. Instr. and Meth. A 581 (2007) 384.

[2] C. Trotta et al., New high spatial resolution portable camera in medical imaging, Nucl. Instr. and Meth. A 577 (2007) 604.

[3] Y. Chou et al., Observations of Cygnus X-1 with the EXITE2 hard X-Ray balloon payload, ApJ. 618 (2005) 856.

[4] A. Breskin et al., Advances in gas avalanche photomultipliers, Nucl. Instr. and Meth. A 442 (2000) 58.

[5] T. Francke et al., Novel position-sensitive gaseous detectors with solid photocathodes, IEEE Trans. Nucl. Sci. 49 (2002) 977.

[6] Y. Giomataris et al., MICROMEGAS: a high-granularity position-sensitive gaseous detector for high particle-flux environments, Nucl. Instr. and Meth. A 376 (1996) 29.

[7] B. Ketzer et al., Performance of triple GEM tracking detectors in the COMPASS experiment, Nucl. Instr. and Meth. A 535 (2004) 314.

[8] F. Sauli, GEM: A new concept for electron amplification in gas detectors, Nucl. Instr. and Meth. A 386 (1997) 531.

[9] M. Inuzuka et al., Gas electron multiplier produced with the plasma etching method, Nucl. Instr. and Meth. A 525 (2004) 529.

[10] A. Ochi et al., A new design of the gaseous imaging detector: Micro Pixel Chamber, Nucl. Instr. and Meth. A 471 (2001) 264.

[11] T. Nagayashi et al., Development of $\mu$-PIC and its imaging properties, Nucl. Instr. and Meth. A 525 (2004) 20.

[12] F. Tokanai et al., Development of gaseous PMT with micropattern gas detector, Nucl. Instr. and Meth. A 610 (2009) 164.

[13] P. Dorenbos et al., Scintillation properties of $\mathrm{Nd}^{3+}$ doped LaF 3 crystals, IEEE Trans. Nucl. Sci. 37 (1990) 119.

[14] T. Yanagida et al., Basic Properties of Ceramic Pr:LuAG Scintillators, IEEE Trans. Nucl. Sci. 56 (2009) 2955.

[15] J. van der Marel et al., $A \mathrm{LaF}_{3}: \mathrm{Nd}(10 \%)$ scintillation detector with microgap gas chamber read-out for the detection of $\gamma$-rays, Nucl. Instr. and Meth. A 392 (1997) 310.

[16] Hamamatsu Photonics K.K.; data sheet; Japan.

[17] H. Kubo et al., Development of a time projection chamber with micro-pixel electrodes, Nucl. Instr. and Meth. A 513 (2003) 94.

[18] R. Orito et al., Development of an ASD IC for the Micro Pixel Chamber, IEEE Trans. Nucl. Sci. 51 (2004) 1337. 